\title{
Dynamic Factorial Structure of Perceived Condition for a Women's Competitive Walker
}

\author{
Takahiro Nakano* and Takahiko Nishijima* \\ *Institute of Health and Sport Sciences, University of Tsukuba \\ taka@stat.taiiku.tsukuba.ac.jp \\ 1-1-1, Tennodai, Tsukuba City, Ibaraki 305-8574 Japan \\ [Received May 26, 2003 ; Accepted November 25, 2003]
}

\begin{abstract}
The purpose of this research is to identify a dynamic factor structure of an athlete's perceived condition fluctuation using a dynamic factor analysis (DFA) and a confirmatory factor analysis (CFA). The subject of the study was an athlete who represented Japan in the women's race walking in the 2002 Asian Games. The subject made records of 15 items concerning perceived conditions, training, and basic lifestyle habits everyday for 714 days on QC sheets for conditions that she created herself. As factor analysis from an exploratory standpoint, a DFA using a maximum likelihood method and an orthogonal rotation by varimax method was applied, and common factors of perceived condition fluctuation was extracted with consideration given to time lag. Furthermore, CFA by structural equation modeling was applied to identify a dynamic factor structure of perceived condition fluctuation. Based on the coefficient of autocorrelation and the coefficient of partial autocorrelation of the variables, it was found best to use avariance-covariance matrix of Lag 1 in DFA. Three factors of weather conditions, sleeping, and getting up were extracted in all lags. However, training factor was extracted in Lag 0 only and perceived condition factor in Lag 1 only. The fit index of the dynamic factor structure model of perceived conditions where five factors were hypothesized was favorable. It was concluded that perceived condition fluctuation is a dynamic factor structure that was influenced from change in time.
\end{abstract}

Keywords: multivariate time-series data, dynamic factor analysis, confirmatory factor analysis, QC Sheet

[International Journal of Sport and Health Science Vol.2, 67-75, 2004]

\section{Introduction}

In order for an athlete to perform at his or her best it is essential to understand accurately and adjust both mental and physical conditions. Preparing to be at one's best condition for an athletic meet of target requires understanding and controlling one's own conditions every day. An athlete's conditions fluctuate due to influences from contents and amount of training, and can be measured daily in terms of items relating to perceived conditions, basic lifestyle habits, and training. For an athlete to be able to understand and control fluctuations of conditions for his or her self, the athlete's structure of condition fluctuation needs to be clarified. In addition, since there is a characteristic that particularity of condition fluctuation becomes stronger if an athlete competes at a higher level, it is necessary to capture the structure of condition fluctuation for each individual (Nakano and Nishijima, 2001; Nishijima et al., 2000).
In this research, particularity of condition fluctuation of athletes is considered and ideas of the single-case study method are applied. In a single-case study method series fluctuations within an individual are treated as the subject (Smith,1988; Barlow and Hersen, 1984; Kazin, 1982; Zaichkowsky, 1979), and evaluation methods using statistical tests are proposed [Yamada, (1999); Yamada, (1998)].

In this study multivariate time series data concerning an athlete's condition fluctuation were acquired by continuously observing and measuring such athlete's conditions using multiple items. The data obtained by using multiple variables are, in many cases, contracted to a small number of factors using a factor analysis and are interpreted. However, many of the methods applied to previous multivariate time series data were based on P-technique where a general factor analysis method was applied with no consideration of serial correlation which is unique to time series data [Nishijima et al., (2000); Kinugasa 
Table 1 Measurement items

\begin{tabular}{|c|c|c|c|}
\hline Domain & Subdomain & Items (Measurement method) & \\
\hline $\begin{array}{l}\begin{array}{l}\text { Subject } \\
\text { condition }\end{array} \\
\end{array}$ & $\begin{array}{l}\text { Perceived } \\
\text { condition }\end{array}$ & $\begin{array}{l}\text { Perceived fatigue (five-scale) }{ }^{*} \\
\text { Emotion instability (five-scale) }\end{array}$ & Perceived physical condition (five-scale) ${ }^{*}$ \\
\hline \multirow{4}{*}{ Living } & Training & $\begin{array}{l}\text { Perceived will to training (five }- \text { scale) } \\
\text { Perceived intensity of training (five-scale)* } \\
\text { Perceived achievement of training (five-scale) } \\
\text { Body weight after last training (up to } 1 / 10 \text { ) }\end{array}$ & $\begin{array}{l}\text { Training fatigue (five-scale) } \\
\text { Training hours (notation for } 24 \text { hours) } \\
\text { Body weight after training (up to } 1 / 10 \text { ) } \\
\text { Body fat after training (up to } 1 / 10 \text { ) } \\
\end{array}$ \\
\hline & Eating & $\begin{array}{l}\text { Satisfaction for breakfast (three-scale) } \\
\text { Satisfaction for dinner }\left(\text { three-scale) }{ }^{*}\right. \\
\text { State of defecation (four-scale) }\end{array}$ & $\begin{array}{l}\text { Satisfaction for lunch (three-scale) } \\
\text { Appetite(five-scale) }\end{array}$ \\
\hline & $\begin{array}{l}\text { Physical } \\
\text { condition }\end{array}$ & $\begin{array}{l}\text { Heart rate when getting up (up to } 1 / 10)^{*} \\
\text { Body weight when getting up (up to } 1 / 10)^{*} \\
\text { Body fat of when getting up (up to } 1 / 10)^{*} \\
\end{array}$ & $\begin{array}{l}\text { Temperature when getting up (up to } 1 / 10 \text { ) } \\
\text { Blood pressure when getting up }\end{array}$ \\
\hline & Sleeping & $\begin{array}{l}\text { Bedtime (notation for } 24 \text { hours) } \\
\text { Sleeping hours (notation for } 24 \text { hours) }\end{array}$ & $\begin{array}{l}\text { Getting up time (notation for } 24 \text { hours) } \\
\text { Level of sound sleep (five-scale)* }\end{array}$ \\
\hline Environment & $\begin{array}{l}\text { Weather } \\
\text { condition }\end{array}$ & $\begin{array}{l}\text { Durations of sunshine (notation for } 24 \text { hours) } \\
\text { Average humidity (up to } 1 / 10)^{*} \\
\text { Maximum air temperature (up to } 1 / 10 \text { ) }\end{array}$ & $\begin{array}{l}\text { Weather } \\
\text { Average temperature (up to } 1 / 10 \text { ) } \\
\text { Minimum air temperature (up to } 1 / 10 \text { ) }\end{array}$ \\
\hline
\end{tabular}

* :Items for statitical data analysis

et al., (2002)]. For this reason, it was not possible to analyze the series fluctuation of factor structure of a phenomenon, such as an athlete's condition fluctuation, that may influence other factors with a time lag. Molenaar (1985) proposed, as a solution to this problem, a dynamic factor analysis (DFA) which is an extended form of a factor analysis method based on P-technique.

In previous research on condition fluctuations, Nishijima et al. (2000) applied a general factor analysis model to series fluctuations and suggested the existence of high-order latent factors that are commonly engaged among fluctuation factors. Nakano, et al. (2001) identified a second-order factor structure of condition fluctuation. Furthermore, Nakano, et al. (2003) pointed out the applicability of dynamic factor analysis (DFA) to condition fluctuation data.

Considering the above, the purpose of this study was to confirm a dynamic factor structure of an athlete's time-series condition fluctuation.

\section{Methods}

\subsection{Subjects}

The subject of the study was an athlete who represented Japan in the women's race walking in the 2002 Asian Games, age twenty-five, height and weight at the beginning of measurement were 154.0 $\mathrm{cm}$ and $46.0 \mathrm{~kg}$. Her athletic career was 13 years by then. She observed and measured her own perceived condition fluctuations continuously for 714 days (102 weeks) from May 1, 2000 to April 14, 2002. Her most prominent result in competition was winning the $20 \mathrm{~km}$ race at the National Athletics Championships of Japan in 2002 with a time of 1 hour, 33 minutes, and 34 seconds.

\subsection{Measurement Method}

Much consideration was given to minimizing the subject's burden in gathering condition data every day for a long period of time as an athlete. Initially there were discussions with the subject to determine what and how many items could be measured continuously through self-observation. In addition, emphasis was given to items that would enhance the subject' s self-control through continuous measurement. We explained to the subject the importance of controlling her own conditions by herself and techniques of self-.

As shown in Table 1, measurement items were primarily classified into subject conditions, life, environment, and natural environment. Based on the definitions by Nishijima et al. (2000), measurement items were composed of those items of perceived body conditions, items of basic lifestyle behaviors including training, and items of natural environment factors. As self-control items for the athlete, heart rate when getting up, temperature when getting up, body weight after training, and items concerning the contents of training were added. Items relevant to eating were: nutrient balance in breakfast, lunch, and dinner; perceived satisfaction concerning amount of food; and appetite for each day.

Measurement items of conditions were finally selected by the subject and recording sheets useful for her were created. Three items in the perceived 
condition domain of the subject conditions were: perceived conditions, perceived fatigue, and emotion instability. In the life domain, there were eight training domain items: perceived will to train, perceived intensity of training, perceived achievement of training, perceived training fatigue, training hours, body weight after training, body fat after training, and body weight after last training. Five eating domain items consisted of: satisfaction for breakfast, satisfaction for lunch, satisfaction for dinner, appetite, and state of defecation. Five awakening domain items were: heart rate when getting up, temperature when getting up, body weight when getting up, body fat when getting up, blood pressure when getting up. Four sleeping domain items included: bedtime, getting up time, sleeping hours, and level of sound sleep. There were six items of weather condition domain in the environment domain, namely: durations of sunshine, average humidity, weather, average temperature, maximum air temperature, and minimum air temperature, where data published by Japan Meteorological Agency were used. The format of recording sheets was adopted from "QC sheets for conditions" described in Nishijima, et al. (1994) and Nishijima, et al. (2000). Data for one week were recorded on one sheet of A4 size paper.

Items concerning time and duration were recorded according to the 24-hour clock system. For each of the three items of satisfaction for dinner, satisfaction for lunch, and satisfaction for breakfast, the subject was required to select an appropriate answer from three variables: (1) little, (2) average, or (3) plenty. Variables for state of defecation were: (1) good evacuation, (2) constipation, (3) diarrhea, and (4) none. Available selections for weather were: (1) clear, (2) cloudy, (3) rain, and (4) snow. All other perceived conditions were given five variables to choose from.

A total of 15 items were used in statistical examination; three from the subject condition domain, 10 from the life domain, and two from the environment domain.

\subsection{Statistical Analysis}

Since the subject did not record any items relevant to training on days when she had no training, there were many missing values for such items. Considering that the data are time series data, missing values were filled in using the linear trend method. Common factors of condition fluctuation were extracted by applying a DFA to the multivariate time series data of 714 days. Based on the result of extraction, a dynamic factor structure of perceived condition fluctuation was identified using structural equation modeling (SEM).

Since multivariate time series data contain serial correlation which is uniquely found in time series data, it is recommended to use, instead of a general factor analysis method, a DFA which is a factor analysis method with consideration given to serial correlation [Kettunen and Ravaja, (2000); Velicer and Plummer, (1998); Molenaar, (1997); Wood and Brown,(1997); Molenaar, (1985)]. The present study used a DFA which aims to identify a dynamic factor structure, although time series factor analysis is recommended as a factor analysis method applicable to multivariate time series data [Buuren, (1997); Toyoda, (1997)].

Up to now, a factor analysis method based on P-technique as proposed by Cattell (1952) is known as a factor analysis method for time series data. A factor analysis method based on P-technique applies a normal factor analysis method to multivariate data where points of time are the subject of observation: therefore, it has a theoretical flaw that no consideration is given to serial correlation of data (Anderson, 1963). A DFA is a method proposed by Molenaar (1985) in which a factor analysis is computed using a lagged variance-covariance matrix in order to solve this problem. A model formula of a DFA is shown below:

$$
v_{t}=\sum_{u=0}^{s} \Lambda_{u} \eta_{(t-u)}+\varepsilon_{t}
$$

where $\mathrm{Z}$ is the observation variable, $\lambda$ is the factor loading, $\eta$ is the latent variable, $\varepsilon$ is the error variable, the suffix t represents time, and the suffix $u$ is the number of lags incorporated. As in the cases of Hershberger, et al. (1994) and Wood \& Brown (1994), a DFA is applied from an exploratory standpoint in this study.

In a DFA, a number of lags to be incorporated in a variance-covariance matrix is determined initially. The number of lags to be considered for this study was determined according to significance of the autocorrelation and the partial autocorrelation coefficient in each variable.

A maximum likelihood method was used as a factor extraction method, and a factor pattern matrix after an orthogonal rotation by varimax method was computed to obtain a simple structure of common factors. The DFA provided an initial solution for each lag. Initial solutions of lags were dealt with individually to perform a factor rotation [Molenaar, (1985)]. Since a correlation matrix between factors is assumed to be a unit matrix in a DFA, an oblique 
Table 2 Descriptive.statitics

\begin{tabular}{llrrrrr}
\hline Domain & Variables & $\mathrm{N}$ & Min & Max & Mean & $\mathrm{SD}$ \\
\hline \multirow{2}{*}{ Subject } & Perceived fatigue & 713 & 1 & 4 & 2.7 & 0.5 \\
& Perceived physical condition & 713 & 1 & 5 & 3.0 & 0.6 \\
& Emotion instability & 713 & 1 & 5 & 2.7 & 0.5 \\
\hline \multirow{6}{*}{ Living } & Perceived will to training & 464 & 2 & 5 & 3.1 & 0.5 \\
& Perceived intensity of training & 463 & 1 & 5 & 2.7 & 1.0 \\
& Perceived achievement of training & 465 & 1 & 5 & 3.0 & 0.6 \\
& Satisfaction for dinner & 714 & 1 & 3 & 2.0 & 0.3 \\
& Heart rate when getting up & 712 & 40 & 76 & 50.2 & 5.5 \\
& Body weight when getting up & 705 & 42.4 & 51.4 & 46.7 & 2.0 \\
& Body fat when getting up & 693 & 19.5 & 29.5 & 23.7 & 2.4 \\
& Bedtime & 714 & 20 & 26 & 22.3 & 0.5 \\
& Sleeping hours & 714 & 2.17 & 9.83 & 6.8 & 1.0 \\
& Level of sound sleep & 711 & 1.5 & 5 & 3.4 & 0.8 \\
\hline \multirow{2}{*}{ Environment } & Durations of sunshine & 714 & 0 & 12.9 & 6.1 & 4.2 \\
& Average humidity & 714 & 21 & 94 & 62.1 & 14.6 \\
\hline
\end{tabular}

Table 3 Autocorrelation.(ACF).and.Partial.autocorrelation.(PACF)

\begin{tabular}{lccc}
\hline Variables & Lag1 & Lag2 & Lag2 \\
& ACF(PACF) & ACF & PACF \\
\hline Perceived fatigue & $0.219^{*}$ & $0.132^{*}$ & $0.088^{*}$ \\
Perceived condition & $0.707^{*}$ & $0.650^{*}$ & $0.300^{*}$ \\
Emotion instability & $0.426^{*}$ & $0.300^{*}$ & $0.146^{*}$ \\
\hline Perceived will to training & $0.125^{*}$ & $0.088^{*}$ & 0.073 \\
Perceived intensity of training & $0.515^{*}$ & $0.442^{*}$ & $0.241^{*}$ \\
Perceived achievement of training & $0.129^{*}$ & 0.070 & 0.055 \\
Satisfaction for dinner & $0.183^{*}$ & 0.042 & 0.009 \\
Heart rate when getting up & $0.199^{*}$ & $0.191^{*}$ & $0.158^{*}$ \\
Body weight when getting up & $0.971^{*}$ & $0.959^{*}$ & $0.288^{*}$ \\
Body fat when getting up & $0.960^{*}$ & $0.948^{*}$ & $0.339^{*}$ \\
Bedtime & $0.253^{*}$ & $0.221^{*}$ & $0.168^{*}$ \\
Sleeping hours & $-0.156^{*}$ & -0.011 & -0.036 \\
Level of sound sleep & $0.494^{*}$ & $0.444^{*}$ & $0.265^{*}$ \\
\hline Durations of sunshine & $0.288^{*}$ & 0.055 & -0.030 \\
Average humidity & $0.488^{*}$ & $0.266^{*}$ & 0.036 \\
\hline
\end{tabular}

rotation cannot be performed [Hershberger, et al., (1994)]. Variables having a factor loading of 0.2 or greater for a factor structure of each lag were referenced to interpret the factors, and names were given. Furthermore, as in the case of a general factor analysis, commonality was considered to indicate the degree of variance of each measurement items being described by a common factor.

A dynamic factor structure of perceived condition fluctuation was hypothesized based on the DFA which was performed on an exploratory standpoint, and it was confirmed using a confirmatory factor analysis (CFA) modeling in SEM. A maximum likelihood method was used for assuming parameters. Variables ( $\mathrm{t}-1)$ with a lag of one day and variables ( $t-0)$ without a lag of one day were used in the dynamic factor structure model. Furthermore, fit indices were compared with those of a factor structure model without lags which uses a similar model. The fit indices used for the model were: GFI and AGFI which show explanation rate of the model to the variance-covariance of data for the model; CFI and NFI, which indicate how far the model is off from an individual model that has the worst fit; and RMSEA, which shows distance between the variance-covariance matrix of the model and that of real model.

Application software SPSS11.0J was used for treating missing values in data, calculation of descriptive statistics values, and calculation of the autocorrelation and the partial autocorrelation coefficient, SAS 8.2 for DFA, and Amos 4.0J for CFA. The statistical significance level was set to be 0.05 universally.

\section{Results}

\subsection{Descriptive Statistics Values}

Table 2 shows descriptive statistics values. The mean value of items that were measured in a five choice method was in a range from 2.7 to 3.4. The mean value of satisfaction for dinner which was measured in a three choice method was 2.0. The maximum value of body weight when getting up was $51.4 \mathrm{Kg}$ and the minimum value was $42.4 \mathrm{Kg}$ with a range of $9.0 \mathrm{Kg}$ and a standard deviation (SD) of $2.0 \mathrm{Kg}$. Values of body fat when getting up showed a similar tendency. Among sleeping domain items, the standard deviation for bedtime and sleeping hours was less than one hour each.

\subsection{Autocorrelation and Partial Autocorrelation Coefficient}

Table 3 shows the autocorrelation and the partial autocorrelation coefficient. For Lag 1, the autocorrelation and the partial autocorrelation coefficient take the same value. All items showed a significant level of autocorrelation coefficient for Lag 
Table 4 Factor.pattern.matrix.after.varimax.rotation.among.variables.without.time.lag

\begin{tabular}{lrrrrr}
\hline Variables & $\begin{array}{c}\text { F1 } \\
\text { Training }\end{array}$ & $\begin{array}{c}\text { F2 } \\
\text { Weather } \\
\text { condition }\end{array}$ & $\begin{array}{c}\text { F3 } \\
\text { Sleeping }\end{array}$ & $\begin{array}{c}\text { F4 } \\
\text { Awakening }\end{array}$ & Communality \\
\hline Perceived will to training & 0.77 & -0.02 & -0.10 & -0.04 & 0.60 \\
Perceived achievement of training & 0.75 & 0.02 & 0.03 & -0.12 & 0.58 \\
Perceived intensity of training & 0.27 & 0.08 & -0.07 & 0.08 & 0.09 \\
Emotion instability & 0.26 & 0.04 & 0.02 & 0.00 & 0.07 \\
Level of sound sleep & $\underline{0.14}$ & 0.07 & 0.02 & 0.09 & 0.03 \\
Perceived physical condition & $\underline{0.13}$ & 0.04 & -0.02 & 0.13 & 0.04 \\
Average humidity & 0.07 & 0.42 & 0.03 & -0.02 & 0.18 \\
Durations of sunshine & -0.03 & -0.32 & -0.04 & 0.12 & 0.12 \\
Perceived fatigue & -0.06 & $\underline{0.15}$ & 0.12 & 0.08 & 0.05 \\
Body fat when getting up & -0.03 & -0.14 & -0.01 & -0.08 & 0.03 \\
Sleeping hours & -0.02 & -0.03 & 0.25 & -0.04 & 0.07 \\
Satisfaction for dinner & -0.01 & -0.02 & -0.05 & 0.02 & 0.00 \\
Body weight when getting up & -0.02 & -0.02 & 0.02 & $\underline{-0.10}$ & 0.01 \\
Bedtime & 0.01 & 0.01 & 0.02 & $\underline{-0.09}$ & 0.01 \\
Heart rate when getting up & 0.03 & 0.10 & -0.01 & 0.03 & 0.01 \\
\hline Contibution of factor & 1.34 & 0.35 & 0.10 & 0.10 & 1.89 \\
Contibution rate of factor (\%) & 8.96 & 2.32 & 0.68 & 0.65 & 12.61 \\
\hline Shadow:Factor loadings equal to or greater than .20. & & & & \\
Underline:They are relatively large although factor loadings less than & .20. & & &
\end{tabular}

Table 5 Factor.pattern.matrix.after.varimax.rotation.among.variables.without.time.lag

\begin{tabular}{lrrrrr}
\hline Variables & $\begin{array}{c}\text { F1 } \\
\text { Sleeping }\end{array}$ & $\begin{array}{c}\text { F2 } \\
\text { Weather } \\
\text { condition }\end{array}$ & $\begin{array}{c}\text { F3 } \\
\text { Perceived } \\
\text { condition }\end{array}$ & $\begin{array}{c}\text { F4 } \\
\text { Awakening }\end{array}$ & Communality \\
\hline Sleeping hours & 0.93 & 0.00 & 0.00 & 0.08 & 0.88 \\
Bedtime & 0.89 & 0.02 & 0.06 & 0.07 & 0.80 \\
Average humidity & -0.01 & 0.75 & 0.10 & 0.10 & 0.58 \\
Durations of sunshine & -0.05 & -0.61 & 0.03 & -0.05 & 0.37 \\
Satisfaction for dinner & 0.02 & -0.10 & 0.04 & 0.06 & 0.02 \\
Perceived fatigue & 0.10 & -0.04 & 0.54 & 0.12 & 0.32 \\
Perceived physical condition & 0.01 & -0.02 & 0.35 & 0.13 & 0.14 \\
Emotion instability & -0.03 & -0.11 & 0.33 & 0.29 & 0.21 \\
Heart rate when getting up & 0.05 & -0.04 & $\underline{-0.07}$ & -0.02 & 0.01 \\
Level of sound sleep & 0.02 & 0.03 & 0.07 & 0.21 & 0.05 \\
Perceived achievement of training & 0.03 & 0.00 & 0.03 & $\underline{0.18}$ & 0.03 \\
Perceived will to training & -0.03 & 0.04 & 0.08 & $\underline{0.08}$ & 0.02 \\
Body fat when getting up & 0.00 & 0.01 & -0.04 & $\underline{-0.07}$ & 0.01 \\
Body weight when getting up & -0.02 & 0.02 & -0.06 & $\underline{-0.06}$ & 0.01 \\
Perceived intensity of training & -0.01 & 0.00 & 0.00 & 0.02 & 0.00 \\
\hline Contibution of factor & 1.68 & 0.96 & 0.57 & 0.24 & 3.44 \\
Contibution rate of factor (\%) & 11.22 & 6.38 & 3.78 & 1.59 & 22.97 \\
\hline Shadow:Factor loadings equal to or greater than .20. & & & & \\
Underline:They are relatively large although factor loadings less than & .20. & & &
\end{tabular}

total variance and were named in descending order of factor contribution: training, weather c onditions, sleeping, and awakening. For the training factor, perceived will to train, p e r c e i v ed achievement of training, perce i v ed i n t e n s i t y of training, and emotion i n s t a b i 1 i t y had a factor loading of 0.2 or greater. Average humidity and durations of sunshine for the weather condition factor and sleeping hours for the sleeping factor, respectively, showed a factor loading of 0.2 or greater. Level of sound sleep and perceived 1. On the other hand, the autocorrelation coefficient for Lag 2 had a significant level in 11 out of 15 items. The partial autocorrelation coefficient for Lag 2 took a significant value in 9 of 15 items with the maximum value of 0.339. Based on these results, DFA was computed using a lagged variance-covariance matrix up to Lag 1.

\subsection{Dynamic Factor Analysis}

Table 4 shows a factor pattern matrix for Lag 0 after an orthogonal rotation by varimax method. Four factors were extracted that explain $13 \%$ of the conditions for the training factor and body weight when getting up and bedtime for the awakening factor, respectively, displayed a factor loading less than 0.2; however, these items were referenced in naming the factors since they showed a relatively high factor loading.

Table 5 shows a factor pattern matrix for Lag 1 after an orthogonal rotation by varimax method. Four factors were extracted and were given a name each: sleeping, weather conditions, perceived conditions, and awakening. Variables that showed a factor loading of 0.2 or greater were: sleeping hours and bedtime for the sleeping factor; average humidity 


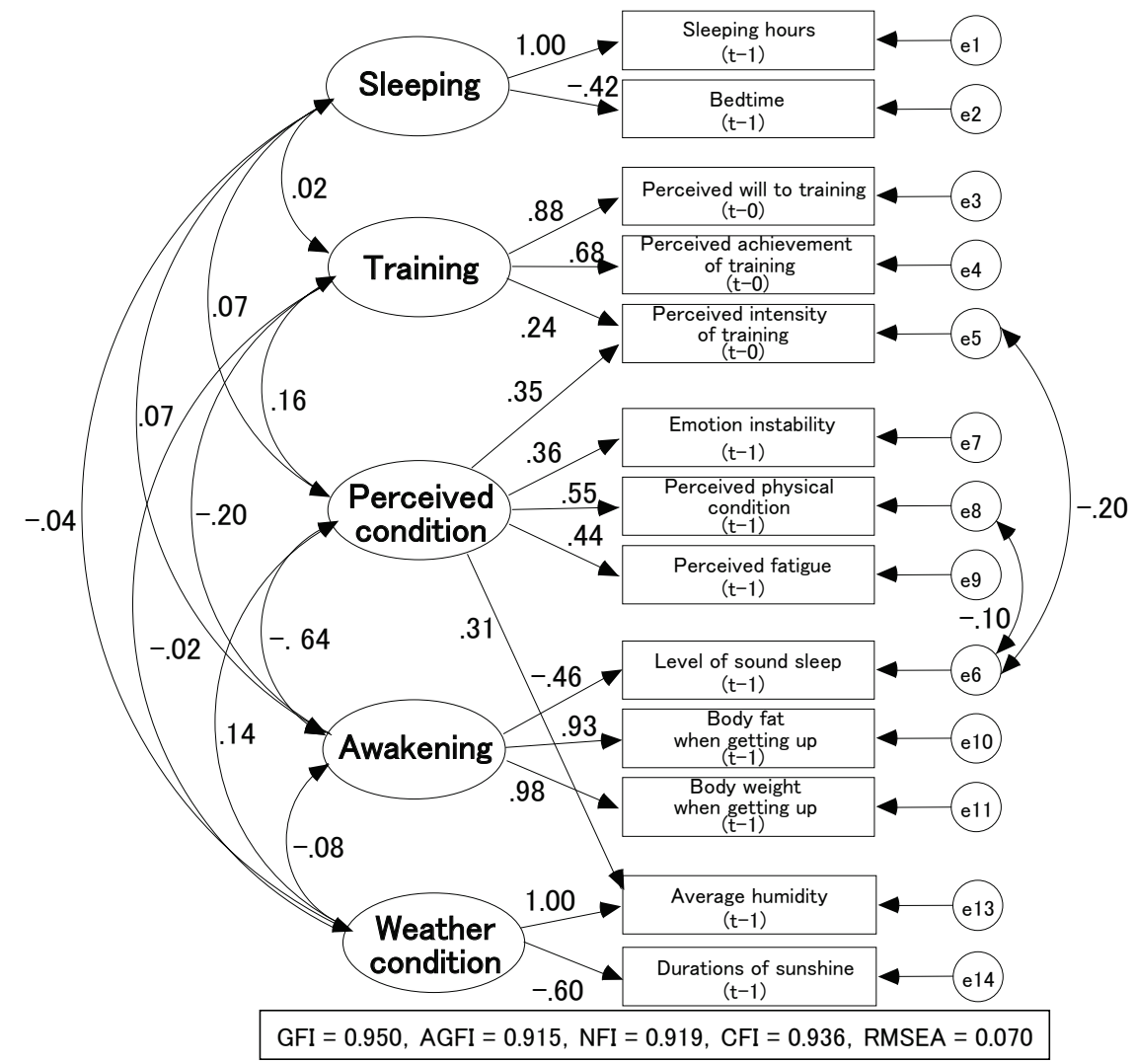

Figure 1 Dynamic factor structure using variables with a lag perceived condition fluctuation. Because a confirmatory factor structure model was built from items referenced for naming of the factors in the DFA, items of satisfaction for dinner and heart rate when getting up were eliminated from the model. Since the training factor was extracted for only Lag 0 as a result of DFA, it was constructed using Lag 0 variables $(\mathrm{t}-0)$ only. On the other hand, the perceived condition factor was extracted only for Lag 1 and was constructed with Lag 1 variables (t-1) only. All other factors, namely the sleeping factor, the awakening factor, and the weather condition factor, were extracted for both lags. However, they were constructed using Lag 1 variables ( $\mathrm{t}-1)$ because all items showed a higher factor loading when Lag 1 was considered. Furthermore, the model and durations of sunshine for the weather condition factor: perceived fatigue, perceived conditions, and emotion instability for the perceived condition factor; emotion instability and level of sound sleep for the awakening factor. Variables that showed a relatively high factor loading, although less than 0.2 , and were referenced in naming the factors were: body fat when getting up and body weight when getting up for the awakening factor.

Three factors, namely the weather conditions, the sleeping, and the awakening, were extracted as common factors in factor pattern matrices for both Lag 0 and Lag 1 . The training factor was extracted as a common factor for Lag 0 whereas the perceived condition factor was extracted as a common factor when Lag 1 was considered. The total percentage of explained variance of the common factors was $13 \%$ for Lag 0, 23\% for Lag 1, and 36\% combined.

\subsection{Dynamic Factor Structure of Perceived Condition Fluctuation}

In order to comfirm a dynamic factor structure, variables (t-1) with a lag of one day and variables $(\mathrm{t}-0)$ without a lag of one day were created for each item. Figure 1 shows a dynamic factor structure of was improved by, firstly, eliminating a path from a factor to a respective variable if the path coefficient was not significant. Because of this criterion, the path from the training factor to level of sound sleep (t-1) was deleted. Secondly, if a new path could be considered content validity based on a adjust index, it was introduced. Because of this criterion, a path was added from the perceived condition factor to perceived intensity of training $(\mathrm{t}-0)$ and another to average humidity (t-1). Thirdly, based on a adjust index, covariance of errors was hypothesized. There were two points that required care when hypothesizing covariance of errors: (1) relation between items could be considered content validity, and (2) items of concern could be verified to have higher-level factors whose relation is recognized. According to this criterion, covariance was hypothesized among errors of the following variables: level of sound sleep ( $\mathrm{t}-1)$, perceived intensity of training $(\mathrm{t}-0)$, and perceived fatigue (t-1). The model after applying the corrections above provided favorable fit indices such as $\mathrm{GFI}=0.950, \mathrm{AGFI}=0.915, \mathrm{NFI}=0.919, \mathrm{CFI}=$ 0.936 , and RMSEA $=0.070$. Thus a valid model was confirmed. 


\section{Discussion}

\subsection{Availability of Dynamic Factor Analysis}

Data gathered by an athlete through observation of his or her daily condition are multivariate time series data. A statistical analysis technique such as a factor analysis has been used for contraction of dimension for multivariate data, however, a general factor analysis method has a theoretical flaw when used for time series data (Anderson, 1963), in some cases a factor analysis method based on P-technique was applied where a multivariate time series data set is considered not as subject multiplied by variable as normally done but as time multiplied by variable [Cattel, (1952)]. Not only did this method have a flaw in its theory, but its analysis result did not provide information unique to time series data. Since it was thought that application of a factor analysis method based on P-technique was inadequate in order to obtain a fluctuation factor structure with consideration of serial dependency among data, a DFA (Molenaar, 1985) was used as it has been recommended in recent years. As an example of a study where a DFA was applied, Hershberger, et al. (1994) examined emotional response patterns of father-daughter and stepfather-stepdaughter dyads and indicated that the dynamic factor structure of emotional response was changed by taking time lag into consideration.

In this study, a DFA was applied based on the program proposed by Wood \& Brown (1994). The purposes of the program described by Wood \&Brown-were to obtain an initial solution of factor analysis and to compare multiple models in terms of fit indices. On the other hand, in this study, as proposed by Hershberger, et al. (1994), from a stand point of exploratory factor analysis, factor structure was obtained while orthogonal rotation by varimax method was used for the initial solution.

The result of a DFA provided observation of fluctuation factors such as the training factor that can be extracted when no time lag is introduced and others such as the perceived condition factor that can be extracted by giving a time lag. It was also possible to speculate by DFA whether items that were influenced by the factors had DFA could have a time lag for the remaining other factors such as the weather condition factor, the sleeping factor, and the awakening factor. By applying a DFA, it was possible to confirm a factor structure with consideration of time lags, which was not possible with a factor analysis based on P-technique. These results indicate that, with consideration given to time lags, dynamic changes of a fluctuation factor structure and changes of items which are influenced by factors can be confirmed by applying a DFA to multivariate time series data which concern perceived condition fluctuations.

The total percentage of explained variance of common factors in a variance-covariance matrix when using Lag 1 variables (t-1) was $36 \%$, which was rather low. The total percentage of explained variance of common factors could be increased by considering Lag 2 variables in the variance-covariance matrix, However, interpretation of the factor structure is expected to become more complex. Nakano, et al. (2003) reports that a prerequisite condition to applying a DFA to an athlete's perceived condition fluctuation data is that a clear dynamic factor structure is indicated. To solve this problem, this study was conducted to confirmed a dynamic factor structure by hypothesizing a dynamic factor structure of condition fluctuation that closely follows the results of a DFA and using a CFA model in SEM. This procedure is a similar manner as in a procedure of factor structure confirmation used in recent years. Consequently, a dynamic factor structure of condition fluctuation was clearly confirmed by a CFA with lagged variables. This result indicates that factors of perceived condition fluctuations are influenced by lagged variables. Thus, it is shown that, by using a DFA to an exploratory standpoint, a dynamic factor structure with consideration given to lagged variables could be confirmed.

\subsection{Dynamic Factor Structure of Perceived Condition Fluctuation}

The confirmed dynamic factor structure showed that five factors that are related to sleeping, training, perceived conditions, awakening, and weather conditions exist by considering time lag of one day. These factors had a structure that comprehensively includeed factors classified by time periods of lifestyle behaviors similar to the result by Nishijima, et al. (2000) and factors similar to the result of Ohba, et al. (1998), who incorporated many variables concerning training. The path coefficient among 
the three factors of training, perceived conditions, and awakening indicated that the three factors fluctuate while keeping in correlation with one another. Particularly, correlation as strong as -0.64 was found between the perceived condition factor and the awakening factor. Of the three factors, the training factor was composed of Lag 0 variables ( $\mathrm{t}-0)$ only and it was found that it was influenced by the perceived condition factor and the awakening factor of the day before.

Variables that were most influenced by the respective factors were: sleeping hours by the sleeping factor, perceived achievement of training by the training factor, perceived conditions by the perceived condition factor, body weight when getting up by the awakening factor, and average humidity by the weather condition factor. It is expected that the subject can perform self-observation and self-control of her conditions by checking conditions with focus on these five items above. Furthermore, while the training factor was composed of Lag 0 variables (t-0) only, other factors were mainly composed of Lag 1variables ( $\mathrm{t}-1)$. From this result, a possibility was speculated that variables relating to training are strongly influenced by other variables conditions of the day before. Since the model as a whole showed favorable fit indices, perceived condition fluctuation that was self-observed through the use of QC sheets for conditions was found to be composed of these five factors.

As expressed above, by using a DFA and a CFA, it is clearly possible to confirm a dynamic factor structure of perceived condition fluctuation with consideration to lagged variables, therefore it is possible to confirm time influence among factors of condition fluctuation.

\section{References}

Anderson, T. W. (1963) The use of factor analysis in the statistical analysis of multiple time series. Psychometrika 28:1-25.

Buuren, S. V. (1997) Fitting ARMA time series by structural equation models. Psychometrika 62(2): 215-236.

Bollen, K. A. (1989) Structural equations with latent variables. Wiley: New York, pp80-318.

Barlow, D. H., and Hersen, M. (1984) Single-case experimental designs: strategies for studying behavior change ( 2 nd ed.). Pergamon: New York, pp67-106.

Cattel, R. B. (1952) Factor analysis: an introduction and manual for the psychologist and social scientist. Harper, New York: pp.102-112.

Hershberger, S. L., Corneal, S. E., and Molennar, P. C. M. (1994) Dynamic factor analysis: an application to emotional response patterns underlying daughter/father and stepdaughter/stepfather relationships. Structural equation modeling 2: 31-52.

Joreskog, K. G. and Lawley, D. N. (1968) New method in maximum likelihood factor analysis. British Journal of Mathematical and Statistical Psychology 21: 85-96.

Joreskog, K. G. (1969) A general approach to confirmatory maximum likelihood factor analysis. Psychometrika 34: 183-202.

Kazin, A. E. (1982) Single-case research designs: methods for clinical and applied settings. Oxford University Press: New York, pp230-337.

Kettunen, J., and Ravaja, N. (2000) A comparison of different time series techniques to analyze phasic coupling: A case study of cardiac and electrodermal activity. Psychophysiology 37: 395-408.

Kinugasa, T., Miyanaga, Y, Shimojo, H., and Nishijima, T. (2002) Statistical evaluation of conditioning for an elite collegiate tennis player using a single-case design. Journal of strength and conditioning research 16(3) : 466-471.

Molenaar, P. C. M. (1985) A dynamic factor model for the analysis of multivariate time series. Psychometrika 50: 181-202.

Molenaar, P. C. M. (1997) Time series analysis and its relationship with longitudinal analysis. International Journal of sports medicine 18: 232-237.

Nakano, T., and Nishijima, T. (2001) Invariance of factorial structure of fluctuation of condition for college women' s swimmer. Japan Journal of test and evaluation of physical education and sports 1: 35-43. (In Japanese with English abstract)

Nakano, T., Nishijima, T., and Yamada, T. (2003) Factor structural analysis of condition fluctuation by dynamic factor analysis. Japan Journal of Physical Education, Health and Sport Sciences 45: 619-630. In print. (In Japanese with English abstract)

Nishijima, T., Kanno, T., Kawamata, N., Kokudo, S., Kuno, S., Tsuchiya, J., Akima, H., Kobayashi, M., Matsumoto, M., Tanaka, K. (1994) Methodology of sport conditioning in soccer team. ICHPER 36th World Congress proceedings: pp177-182.

Nishijima, T., Nakano, T., and Yamada, T. (2000) Statistical analysis on fluctuation of perceived physical and mental condition using single-case study design. Japan Journal of Physical Education, Health and Sport Sciences 45: 619-630. (In Japanese with English abstract)

Ohba, K., Shibuya, T., Nishijima, T., Nagai, J., and Wada, N. (1998) A study of the control system in condition for the woman long distance runner. Research quarterly for athletics 35: 36-44. (In Japanese with English abstract)

Smith, R. E. (1988) The logic and design of case study research. The sport psychologist 2: 1-12.

Toyoda, H. (1997) Time series factor analysis model: factors generated by autoregression and moving average process. Sociological theory and methods 12: 1-14.

Velicer, W. F., and Plummer, B. A. (1998) Time series analysis in historiometry: A comment on Simonton. Journal of personality 66: 476-486.

Wood, P., and Brown, D. (1994) The study of intraindividual differences by means of dynamic factor models: Rationale, Implementation, and Interpretation. Psychological Bulletin 116: 166-186.

Yamada, T. (1998) Introduction of randomaization tests as methods for analyzing single-case data. Japanese Journal of 
Behavior Analysis 13(1): 44-58. (In Japanese with English abstract)

Yamada, T. (1999) Application of statistical tests for single-case data: Power comparison between randomaization test and C statistic. Japanese Journal of Behavior Analysis 14(2): 87-98. (In Japanese with English abstract)

Zaichkowsky, L. D. (1979) Single case experimental designs and sport psychology research. Psychology of motor behavior and sport: pp171-179.

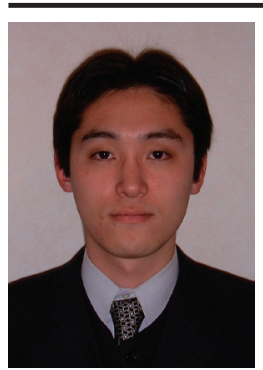

Name:

Takahiro Nakano

\section{Affiliation:}

Researcher(COE), Doctoral Program in Health and Sport Sciences, University of Tsukuba

\section{Address:}

1-1-1 Tennodai Tsukuba City, Ibaraki 305-8574 Japan

Brief Biographical History:

1998- Doctoral Program in Health and Sport Sciences, University of Tsukuba

2003- Received Ph.D at Unversity of Tsukuba

2003- Researcher(COE), Doctoral Program in Health and Sport

Sciences, University of Tsukuba

Main Works:

- "Factor structural analysis of condition fluctuation by dynamic factor analysis"Japan Journal of Physical Education, Health and Sport Sciences, Vol.48(4). 369-381. July. (2003)

Membership in Learned Societies:

- Japan Society of Physical Education, Health and Sport Sciences

- Japan Society of Physical Fitness and Sports Medicine

- American College of Sports Medicine 